\title{
Suicide risk code (CRS): a suicide prevention program in Catalonia
}

S.P. Gomes Da Costa ${ }^{1}$, N. Verdolini ${ }^{2}$, E. Sole ${ }^{3}$, A. Gimenez ${ }^{1}$, G. Anmella ${ }^{1}$, E. Vieta ${ }^{2}$, I. Pacchiarotti ${ }^{2}$.

1 Hospital Clinic, Psychiatry - Clinical Institute of Neuroscience, Barcelona, Spain.

2Hospital Clinic- University of Barcelona- IDIBAPS- CIBERSAM, Bipolar Disorder Unit- Institute of Neuroscience, Barcelona, Spain.

3Hospital Clinic- University of Barcelona- IDIBAPS- CIBERSAM, Perinatal Psychiatry-Institute of Neuroscience, Barcelona, Spain.

\section{INTRODUCTION}

Suicide is one of the leading causes of death in young people and is especially prevalent in patients with psychiatric conditions.

In Catalonia a protocol (Suicide Risk Code) was settled in 2014 to manage cases of suicide attempts. The program is divided in 3 phases: 1) alert and activation, 2) proactive telephone and face-to-face followup from specialized health services and 3) comprehensive preventive health monitoring.

\section{OBJECTIVE}

Describe the clinical characteristics of patients visited in the emergency room for a suicide attempt and included in a suicide prevention program in Catalonia.

\section{METHODS}

A retrospective study was conducted reviewing the clinical and suicide attempt characteristics of the patients entering the program from 2014 to 1 of November of 2017.

\section{CONCLUSIONS}

$\checkmark 15 \%$ of the patients had no previous psychiatric history.

$\checkmark$ The most common diagnosis were: 1. Major Depresive Disorder $\mathrm{NE}$, 2. Substance Use Disorder, 3. Adjustment disorder and 4. Personality disorder

$\checkmark$ The most common type of suicide attempt was intoxication in $84 \%$.

$\checkmark 67 \%$ of the patients attended the follow up visit, and the protocol allowed a faster access to the mental health visit, with an average waiting time of 6 days.

According to previous literature an important amount of people committing suicide attempts have psychiatric history, but the number of people without any previous diagnosis is also alarming. More studies describing the clinical characteristics and results of this type of interventions are needed to evaluate and improve it.

\section{RESULTS}

During the designated period of time, 365 patients from the Hospital Clínic (Barcelona) area of hospitalization were included in this protocol.

\section{SAMPLE}

- 365 patients,

- 218 (59.7\%) females,

- 44.9 years old of mean age.

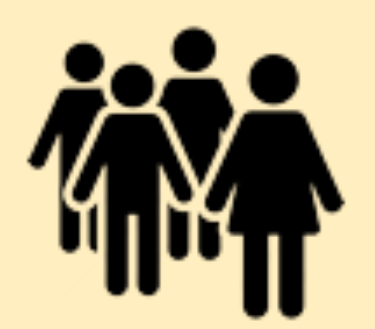

\section{CLINICAL FEATURES}

- No previous psychiatric history: $15 \%$

- At least one psychiatric diagnosis: $85 \%$

- Dual diagnosis (Substance use disorder

+ other psychiatric diagnosis) : $20 \%$

1. Major Depresive Disorder NE

2. Substance Use Disorder

3. Adjustment disorder

4. Personality disorder

5. Others

(Anxiety disorder, Recurrent depression, Disthymia, Acute reaction, Autism, Esquizofrenia, Bipolar Disorder, Major depression 1st episode, OCD, PTSD)

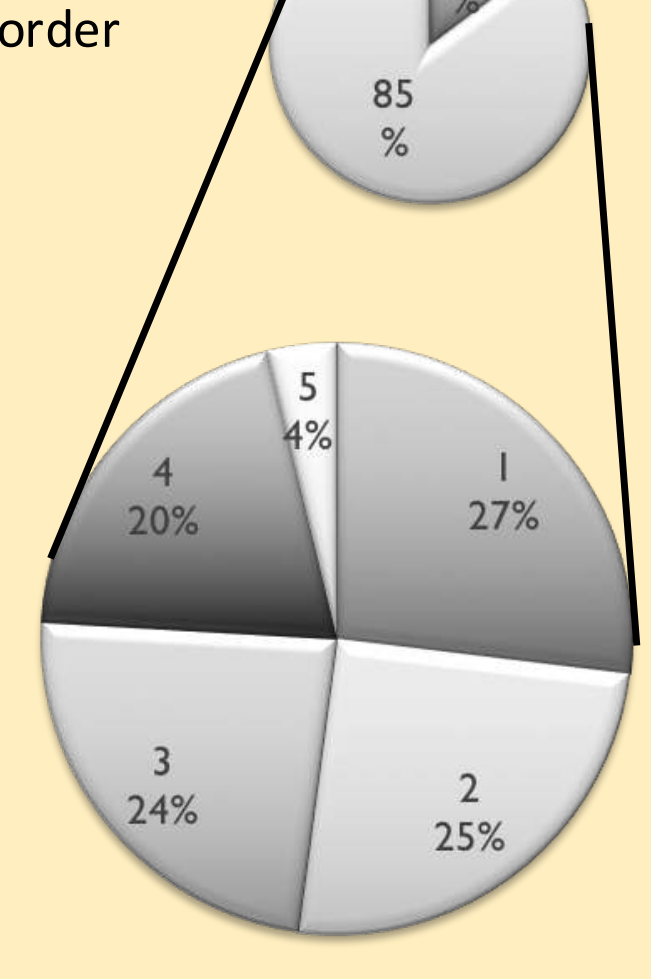

$\square I \square 2 \square 3 \square 4 \square 5$

\section{TYPE OF SUICIDE ATTEMPT}

Suicide attempt: $74 \%$

Suicide ideation: $26 \%$

Suicidal planning: $5 \%$.

Type of suicide attempt:

- Intoxication: 84\%

- Others (Cutting, Jumping from high place, Hanging, Drowning, Other): $16 \%$

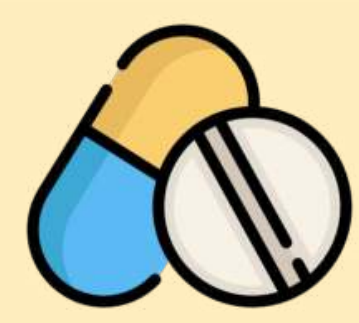

\section{FOLLOW UP}

From the total of patients, $67 \%$ attended the follow up visit.

Average days between the activation of the CRS and the follow-up visit : 6 days

\section{REFERENCES:}

1. American Psychiatric Association Practice Guideline for the Assessment and Treatment of patients with Suicidal Be havior. Supplement of the American J. Psychiatry. Vol 160, no 11, Nov 2003.

2. The Harvard Medical School Guide to suicide assessment and intervention. Douglas Jacobs. Ed. Jossey-Bass Pub. $S$ Francisco USA, 1999

3. Comprehensive Textbook of Suicidology. R: Maris, A. L. Berman, M. M. Silverman. Guilford Press. New York, 2000

4. The international handbook of Suicide and Attempted Suicide. K. Hawton, K. Van Heeringeng. Wiley. England. 2000

5. Prevención de las conductas suicidas y parasuicidas. J Bobes, J.C. Gonzalez, P. Saiz. Masson, Barcelona 1997

6. The suicidal patient. J. Chiles, K.Strosahl. American Psychiatric Press.Washington, 1995

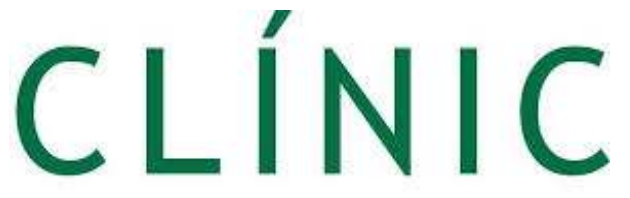

B A R C E L O N A Hospital Universitari 